\title{
How accurate and statistically robust are catalytic site predictions based on closeness centrality? Eric Chea ${ }^{1}$ and Dennis R Livesay*2
}

Address: ${ }^{1}$ Department of Biological Sciences, California State Polytechnic University, Pomona, CA 91768, USA and ${ }^{2}$ Department of Computer Science and Bioinformatics Research Center, University of North Carolina at Charlotte, Charlotte, NC 28223, USA

Email: Eric Chea - erc2009@med.cornell.edu; Dennis R Livesay* - drlivesa@uncc.edu

* Corresponding author

Published: II May 2007

BMC Bioinformatics 2007, 8:153 doi:10.1/86/147|-2105-8-153
Received: II December 2006

Accepted: II May 2007

This article is available from: http://www.biomedcentral.com/I47I-2/05/8/I53

(c) 2007 Chea and Livesay; licensee BioMed Central Ltd.

This is an Open Access article distributed under the terms of the Creative Commons Attribution License (http://creativecommons.org/licenses/by/2.0), which permits unrestricted use, distribution, and reproduction in any medium, provided the original work is properly cited.

\begin{abstract}
Background: We examine the accuracy of enzyme catalytic residue predictions from a network representation of protein structure. In this model, amino acid $\alpha$-carbons specify vertices within a graph and edges connect vertices that are proximal in structure. Closeness centrality, which has shown promise in previous investigations, is used to identify important positions within the network. Closeness centrality, a global measure of network centrality, is calculated as the reciprocal of the average distance between vertex $i$ and all other vertices.
\end{abstract}

Results: We benchmark the approach against 283 structurally unique proteins within the Catalytic Site Atlas. Our results, which are inline with previous investigations of smaller datasets, indicate closeness centrality predictions are statistically significant. However, unlike previous approaches, we specifically focus on residues with the very best scores. Over the top five closeness centrality scores, we observe an average true to false positive rate ratio of 6.8 to I. As demonstrated previously, adding a solvent accessibility filter significantly improves predictive power; the average ratio is increased to 15.3 to $\mathrm{I}$. We also demonstrate (for the first time) that filtering the predictions by residue identity improves the results even more than accessibility filtering. Here, we simply eliminate residues with physiochemical properties unlikely to be compatible with catalytic requirements from consideration. Residue identity filtering improves the average true to false positive rate ratio to 26.3 to I. Combining the two filters together has little affect on the results. Calculated $p$-values for the three prediction schemes range from $2.7 \mathrm{E}-9$ to less than $8.8 \mathrm{E}-134$. Finally, the sensitivity of the predictions to structure choice and slight perturbations is examined.

Conclusion: Our results resolutely confirm that closeness centrality is a viable prediction scheme whose predictions are statistically significant. Simple filtering schemes substantially improve the method's predicted power. Moreover, no clear effect on performance is observed when comparing ligated and unligated structures. Similarly, the $\mathrm{CC}$ prediction results are robust to slight structural perturbations from molecular dynamics simulation. 


\section{Background}

The accurate and robust prediction of protein functional sites from sequence and/or structure remains an open problem in bioinformatics [1]. Despite the limitations of current methodologies, several sequence and structurebased approaches have recently become popular [2]. Most of these approaches rely on an underlying multiple sequence alignment and attempt to uncover some type of feature conservation therein [3] (i.e. residues that are conserved across the alignment [4-6]). Arguably, evolutionary tracing has become the most widely used method for computational prediction of protein functional sites [7]. The Evolutionary trace (ET) approach begins with an alignment and corresponding phylogeny. The method searches for all alignment positions that recapitulate the overall phylogeny. While ET is fundamentally a sequencebased scheme, the standard application of the approach uses structural clusters of trace residues to identify functional regions [8-10]. Several other related methods that rely on an underlying alignment plus representative structure have proven useful as well [11-14]. Conversely, we have introduced a phylogenetic motif-based method that is similar in spirit to ET, although it is specifically designed to rely solely on sequence information [15-17].

The literature also contains a host of functional site prediction strategies that are explicitly designed to not rely on a phylogeny [18]. These approaches are useful when too few sequences are available to generate a representative description of familial diversity. While their theoretical foundations vary considerably, most rely solely on structure or a structure + alignment combination. For example, Gutteridge et al. recently developed a neural network approach to predict catalytic sites [19]. Catalytic sites are defined by residues directly involved in the enzyme-mediated reaction mechanism, which generally constitute a subset of all functional residues. The neural network input of Gutteridge et al. includes both structural and alignment descriptors, and is able to correctly predict the active site in $>69 \%$ of the cases examined. The ability to rigorously benchmark the approach is based on comprehensive databasing and exhaustive manual curation of catalytic residues from the literature [20] by the same group. This tour de force has led to the Catalytic Site Atlas (CSA) [21], which contains approximately 600 different proteins with experimentally validated catalytic residues.

Other common catalytic site prediction methods are based on Poisson-Boltzmann continuum electrostatic theory [22]. Elcock has observed that functional residues tend to have increased electrostatic strain energy [23], meaning that stabilization occurs on mutation. While the approach utilizes sophisticated Poisson-Boltzmann continuum theory, the underlying rationale is based on straightforward evolutionary arguments. The naïve description of protein evolution is that nature solely optimizes structural stability at each residue. However, catalytic and other important residues have functional constraints imposed upon them, meaning that while mutation might be stabilizing, it can occur at the expense of functional proficiency. The detangling of stability and functional evolutionary pressures is examined more thoroughly by Cheng et al. using all-atom protein design [24]. Analogous to the electrostatic strain energy approach, the THEMATICS approach uses Poisson-Boltzmann-based pKa calculations to look for residue titration curves that do not follow Henderson-Hasselbalch [25]. The method looks for titration curves of partially charged residues that are flat over a wide $\mathrm{pH}$ range. Similarly, we have demonstrated that a large pKa shift from the null model (aqueous) value can be indicative of catalytic residues [26,27]. However, the prediction accuracy of this approach is lessened because many structurally important residues (i.e. residues involved in a salt bridge) also have significant pKa shifts.

Network models have also been used with success in predicting protein functional and/or catalytic residues. Instead of representing protein structures as a Cartesian collection of atoms, network models recast protein structures as topological graphs [28-31]. The most common of these methods are based on protein structure contact maps, where each vertex of the graph represents an $\alpha$-carbon and edges connect vertices within some distance cutoff (generally 6-9 $\AA$ ). Once the graph is complete, a variety of topological metrics can be used to predict functional residues from it, including: centrality [32,33], valency [32] and sub-graph conservation [34]. Despite growing consensus concerning the utility of these methods, a robust assessment of their prediction accuracy remains to be completed. Amitai et al. [32], Thibert et al. [33] and del Sol et al. [35] examine the ability of residue centrality to predict catalytic and/or functional sites within datasets of 178,128 and 46 proteins, respectively. The results from these studies are encouraging. Moreover, they show that combining centrality within other metrics improves predictive power. For example, Amitai et al. demonstrates that combining centrality with solvent accessibility substantially improves accuracy, whereas both Amitai et al. and Thibert et al. demonstrate that including residue conservation improves results.

In this report, we investigate the accuracy and statistical significance of closeness centrality (CC) functional residue predictions, which has previously been shown to be the best of several different network centrality scores (i.e. valency, betweenness, etc.) [32,33]. Primarily, our investigation is based on SCOP [36] superfamily-filtered protein chains (which represents 283 unique SCOP superfamilies) from the CSA. Based on observed accuracies, CC 
is demonstrated to be a viable prediction scheme. Our results are inline with previous investigations, but are more significant due to dataset size and composition since we control for structural redundancy. A second distinction of this work is that instead of focusing on the entire range of true to false positive rates, as done by previous investigations, we concentrate on the very best CC scores. By focusing only on the top five scoring residues, we are able to evaluate the ability of the model to provide insight that provides a reasonable number of experimentally testable predictions. In all cases, our predictions correspond to false positive rates below $1.6 \%$. The performance of the method is improved substantially by considering only residues that are not completely inaccessible to solvent. We further demonstrate that filtering the predictions based solely on amino acid identity substantially improves predictive power even more than filtering by solvent accessibility.

\section{Theoretical background}

Throughout this report, the vertices within each graph correspond to $\alpha$-carbons. Edges connect two $\alpha$-carbons within $8.5 \AA$ of each other. While slightly less complicated than methods based on all-atom pair distances, the simpler model results in a noticeable computational speedup that significant when analyzing a dataset the size of ours. A cursory comparison of the two networks indicates that the resultant predictions are qualitatively similar (results not shown). The common threshold of $8.5 \AA$ is used because it best approximates the average sidechain size. Closeness centrality (CC), a global centrality metric, is used to determine how critical each vertex (residue) is in maintaining the small-world behavior of the graph. CC is calculated by:

$$
\mathrm{CC}_{\mathrm{i}}=\frac{N_{p}}{\sum_{j} L_{i j}}
$$

where $N_{p}$ is the total number of vertices in the graph and $\mathrm{L}_{\mathrm{ij}}$ is the shortest path (geodesic distance) between vertices $i$ and $j$. The shortest path is simply the minimum of all possible paths between residues $i$ and $j$. As normally done in protein structure networks, edges are not weighted, making the shortest path simply an integer count of the number of edges separating $i$ and $j$. It should be noted that $\mathrm{N}_{\mathrm{p}}$ (a constant within each protein) has no effect on our observed results since we are only using $\mathrm{CC}$ to rank the residues, meaning the inverse of shortest path sum solely establishes which residues are ultimately predicted. Nevertheless, we employ CC here to be consistent with previous investigations.

\section{Results and discussion \\ Probability density functions}

Mapping CC to structure clearly indicates that residues with high centralities are most likely to occur within the protein core. As is the case in the three examples shown in Fig. 1, catalytic residues frequently do not correspond to the most central residues. Nevertheless, Fig. 2a indicates that there is clear discrimination between the CC probability density functions (PDFs) of catalytic and noncatalytic residues. The data plotted within in Fig. 2 is taken from 283 structurally unique protein chains; meaning no two proteins from a single SCOP superfamily are included. This translates to 96,280 noncatalytic residues and 844 catalytic residues. The PDFs describing datasets parsed by SCOP family (423 proteins) and $80 \%$ pairwise sequence identity (568 proteins) are virtually identical to those shown. The average CC values for the catalytic and noncatalytic residues are 0.19 and 0.16 , respectively. While Fig. 2a suggests that the most extreme CC scores are not likely to be catalytic, catalytic residues are, on average, more central than noncatalytic residues. A two-sample ttest resolutely confirms that the discrimination between the means is statistically significant $(\mathrm{t}=2.0 ; \mathrm{p}=1.6 \mathrm{E}-73$; sample size $=7,372)$. Nevertheless, there is appreciable overlap (59.5\%) between the two PDFs.

Going further, Fig. $2 \mathrm{~b}$ compares the PDFs of residues from three accessibility levels to the catalytic residue PDF. The three accessibility levels roughly correspond to the third most buried, middle third and third most exposed residues within the parsed dataset. At each accessibility level, the catalytic residue PDF has a statistically significant increase within its mean value (see Table 1). As discussed above, this result is slightly counterintuitive because the most buried (and thus, most central) residues frequently are not catalytic. Rather, this result demonstrates that catalytic residues are, on average, more central than the top third most buried residues. Again, this result confirms the earlier observations of Amitai. Yet, caution should be exercised when drawing far-reaching conclusions based on this analysis due to the considerable overlap between the distributions. This is especially true in the case of the buried residues, which has $85 \%$ overlap with the catalytic PDF.

\section{Assessing prediction accuracy of top closeness centrality scores}

As stated above, several investigations have examined the prediction accuracy of global centrality metrics; however, none of the previous investigations are on the scale of this report. Nor, have any rigorously controlled for structural redundancy as we do here. Of the previous reports, the largest dataset investigated is 178 proteins [32], which (unlike ours) contained redundant structural folds. Previous investigations use Receiver Operating Characteristic 


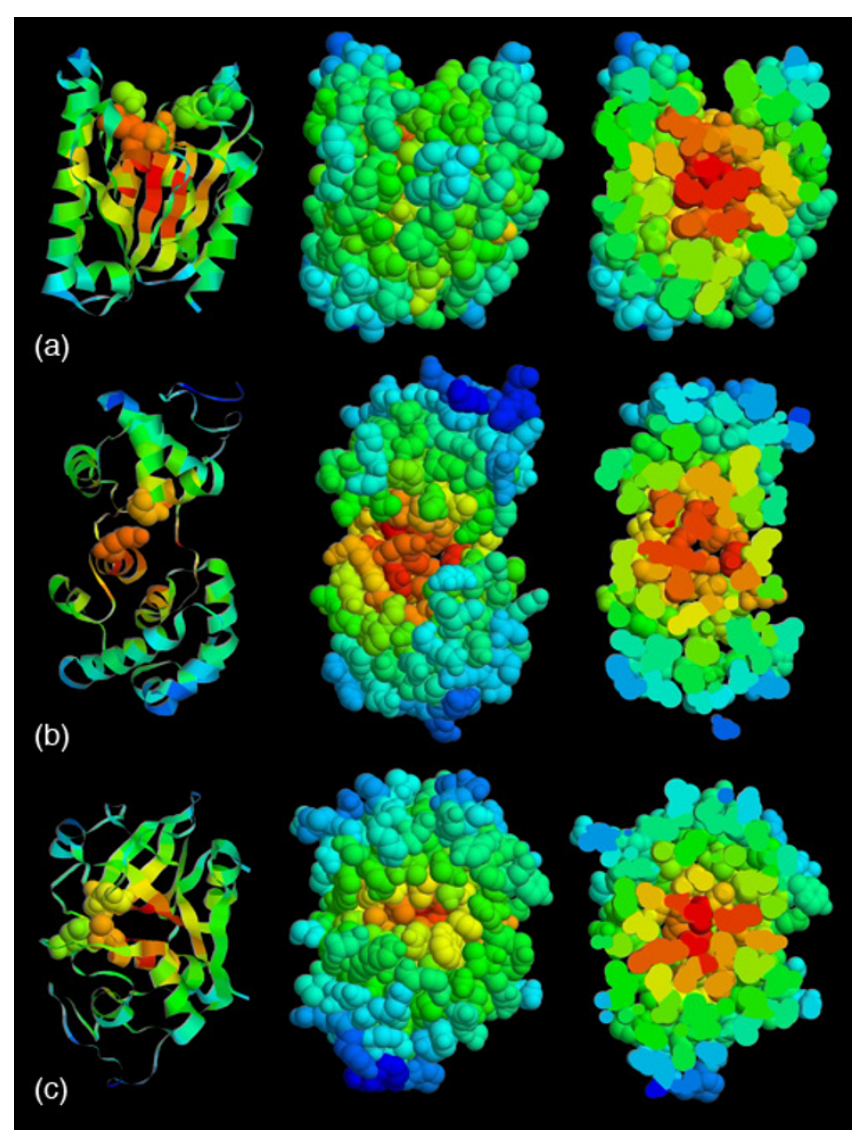

Figure I

Closeness centrality is mapped to structure for: (a) P. purpurogenum Acetylxylan esterase (IBS9), (b) e. coli Endonuclease III (2ABK) and (c) C. papaya papain (9PAP). In all three cases, the most central residues occur near the center of the structure, which do not correspond to the catalytic residues. In each column, red indicates the most central residues; whereas blue indicates the least. In the column on the left, catalytic residues are shown in spacefill. In the center column, all atoms are displayed; in the column on the right, the structure has been sliced in half to highlight the most central residues within the hydrophobic core.

(ROC) plots to examine the balance between true and false positive rates over the entire relationship continuum. A false positive rate greater than $9 \%$ is commonly considered; Thibert et al. routinely consider false positive rates $\sim 20 \%$. In this report, we only consider the top N predictions, where $\mathrm{N}$ equals $1-5$. From a pure prediction point of view, one wants to simultaneously balance sensitivity and specificity. However, when considering experimental realities, we believe our approach has the more relevance because it is less likely to result in huge numbers of false positives that are intractable to test within the lab. The corresponding false positive rate of our predictions is always below $1.6 \%$. Over the entire curve, our ROC plots are virtually superimposable to those within Thibert et al. (results not shown). Unfortunately, a direct comparison to the results within Amitai et al. is impossible since they only provide ROC plots for an integrated sequence conservation/centrality approach. An example ROC plot is provided in Additional file 1.

On average, there are $\sim 5$ catalytic residues per protein within the CSA. We use this value as an appropriate upper bound for the number of predictions per protein to consider. The prediction number threshold, $\mathrm{T}_{\mathrm{np}}$, is defined in order to limit the number of catalytic predictions. At $\mathrm{T}_{\mathrm{np}}=$ 1 , only the site with the top CC score is put forth as a prediction; at $\mathrm{T}_{\mathrm{np}}=2$, the top two are put forth, and so on. However, the actual number of predictions is routinely greater than $\mathrm{T}_{\mathrm{np}}$ due to ties. Consequently, $\mathrm{T}_{\mathrm{np}}$ is scaled between one and five, and the corresponding average number of predictions per protein is 1.3, 2.4, 3.6, 4.6 and 5.7. The circles in Fig. $3 \mathrm{a}$ plot the accuracy (accuracy $=$ number of correct catalytic residue predictions/total number of catalytic residue predictions) against the average number of predictions per protein for each of the three datasets investigated here. The accuracy deviates very little over the full range of $\mathrm{T}_{\mathrm{np}}$; the average and standard deviation are $5.7 \%$ and $0.4 \%$, respectively. When using the common definition of accuracy, (true positives + true negatives)/total number residues, the accuracy corresponds to $98.2 \%$. However, the latter metric provides litthe useful information as the number of true negatives skews it so heavily. (Note: unless otherwise specified, all reported statistics are from the SCOP superfamily parsed dataset.)

The accuracies described above are from a collapsed dataset of all residues contained within the dataset. Alternately, one may wish to evaluate each protein independently and then average the accuracy values over the total number of proteins contained with the dataset. In each case, only the top $T_{n p}$ values per protein are considered, the sole difference is how the final average is determined. Below, statistical significance of the methods is demonstrated by calculating p-values from the binomial distribution, which assume independence of trials. However, the assumption of independence is clearly incorrect as residues are connected via the protein chain. Since this assumption is equally wrong in both cases, we chose to primarily focus on the simpler method of averaging across the collapsed dataset. Nevertheless, Table 2 also lists accuracies calculated on the per protein basis. In all cases, the two values are qualitatively similar; however, per protein values are somewhat reduced. Nevertheless, they are still better than the random expectation. Curiously, there is a large variability across the per protein distribution; the standard deviation is $\sim 10 \%$. The two accuracy calculations are statistically equivalent when using the standard deviation of the per protein values as 

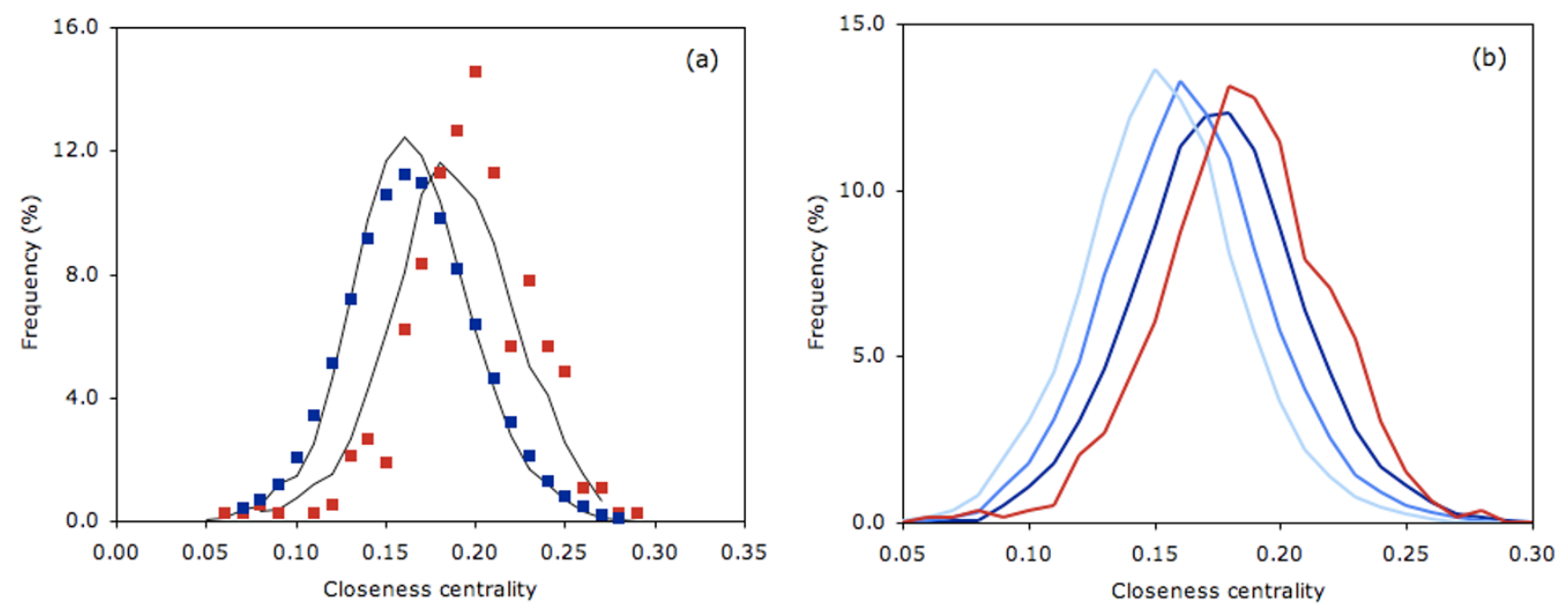

\section{Figure 2}

(a) Probability density function of the closeness centrality scores for all catalytic (red) and all noncatalytic (blue) residues. The distributions are scaled such that the integral of each is equal to one. The solid line trend line is simply a running average meant to help guide the eye. The average values for the two distributions are 0.16 and 0.19 , which is a statistically significant distinction (for a two-sample t-test: $t=2.0 ; p=1.6 E-73$; sample size $=7,373$ ). The overlap of the two distributions across the histogram of sampled data is $59.5 \%$. (b) Probability density functions for all catalytic sites (red line) compared to the distributions of all at three different solvent accessibility levels. Each distribution is scaled such that its integral is one. Statistics describing the distribution comparisons are provided in Table I.

an error estimate; this result is maintained throughout this report. Moreover, the ratio of the collapsed to per protein accuracies is also relatively consistent.

As stated above, p-values are calculated to test the statistical significance of the hypothesis that CC predictions are better than random (Table $2 \mathrm{a}$ ). The binomial distribution is used since there are a finite number of trials and the outcome of each is binary. Eq. 2 provides the formula for calculating the p-values.

$$
P(X>k)=\sum_{k}^{n}\left(\begin{array}{l}
n \\
k
\end{array}\right) p^{k}(1-p)^{n-k} \text {, where }\left(\begin{array}{l}
n \\
k
\end{array}\right)=\frac{n !}{k !(n-k) !}
$$

In Eq. 2, $n$ is equal to the number of predictions put forth, $k$ (in the first iteration of the sum) is equal to the number of correct predictions, and $p$ represents the random (null) probability. Each step of the sum is calculated from the binomial distribution (binomdist) function within Microsoft Excel. Notice that the p-values decrease monotonically (see Additional file 2), despite that the fact that the relative accuracies are not monotonic. In fact, as it is demonstrated below, relative accuracies generally decrease as a function of $\mathrm{T}_{\mathrm{np}}$. Nevertheless, $\mathrm{p}$-values indicate that the results become more statistically significant at larger $\mathrm{T}_{\mathrm{np}}$ values. This apparent contradiction highlights the true meaning of a p-value. A p-value is the statistical likelihood of the null hypothesis being true. It is not an accuracy of the method. The smaller the p-value is, the more significant the observed results are. However, statistical signifi-

Table I: Comparison of accessibility to catalytic residue distributions.

\begin{tabular}{ccccccc}
\hline Distribution' & Average & Std. Dev. & Number residues & t-value $^{2}$ & p-value $^{\text {Percent overlap }^{3}}$ \\
\hline Catalytic & 0.19 & 0.03 & 844 & --- & --- & --- \\
Buried & 0.18 & 0.05 & 6,310 & 2.0 & $1.5 \mathrm{E}-21$ & 84.9 \\
Intermediate & 0.16 & 0.05 & 5,818 & 2.0 & $5.6 \mathrm{E}-58$ & 70.4 \\
Exposed & 0.15 & 0.05 & 6,000 & 2.0 & $1.2 \mathrm{E}-101$ & 58.7 \\
\hline
\end{tabular}

I Accessibility is coarse-grained into three levels, which roughly correspond to the third most buried, middle third and third least buried (exposed) residues. ${ }^{2}$ Comparison of each accessibility-filtered PDF to the catalytic PDF; t-value and p-values are from a two-sample T-test comparing each of the accessibility PDFs to the catalytic residue PDF. ${ }^{3}$ Percent overlap compares each accessibility level distribution to the catalytic residue distribution, which is calculated over the histogram of sampled data. 

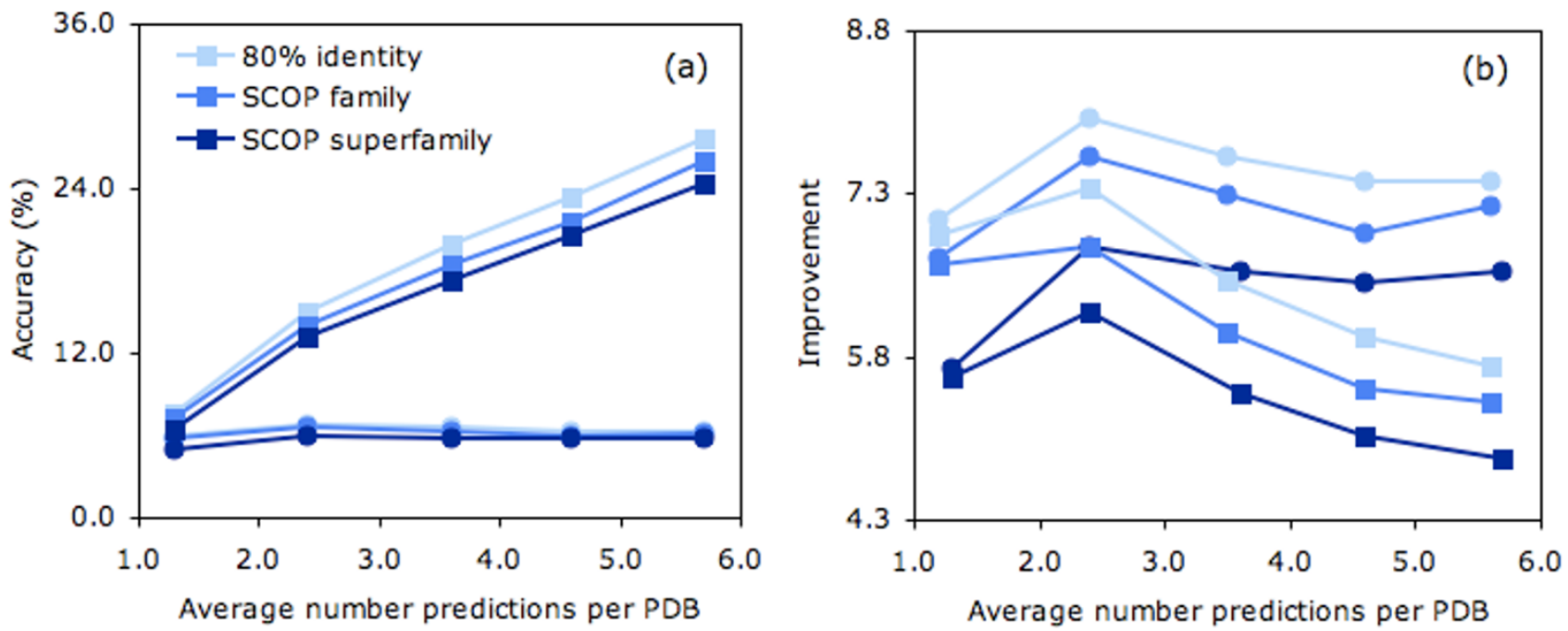

\section{Figure 3}

(a) The accuracy for all predictions (circles) and the percentage of PDBs with one correct prediction (squares) is plotted for the three datasets investigated. (b) Improvement normalizes the observed accuracies in (a) by the random expectation.

cance is intimately related to the number of observations. The more observations of a given difference between an observed and null probability, the more significant it is. The number of predictions put forth put forth at each level increases substantially, whereas the accuracy is only slightly diminished, which is why p-values monotonically decrease as a function $\mathrm{T}_{\mathrm{np}}$.

The improvement is plotted in Fig. 3b in order to normalize the observed percentages by the random expectation. Improvement is defined as the ratio of observed accuracy to random expectation. The random expectation is simply calculated as the percentage of catalytic to all sites within the dataset, meaning each site has an equal chance of being catalytic. While not overwhelming, the observed accuracies $(\sim 6 \%)$ are substantially greater than the null model $(0.9 \%)$. The average improvement over $\mathrm{T}_{\mathrm{np}}=1-5$ is $6.4 \%$ (standard deviation $=0.4 \%$ ). The false positive range in Fig. $3 a-b$ is $0.4-1.6 \%$. The false positive rate is calculated as the number of incorrect predictions divided by the total number of noncatalytic residues. True positive rates (number correct divided by the total number of catalytic residues) range from $2.1-11.0 \%$.

While the circles in Fig 3a correspond to overall accuracies, the squares describe the number of proteins with at least one correct prediction per protein. The near linear increase is trivially expected since the number of proteins with at least one correct should increase with the total number of predictions. However, after normalizing for the random expectation in Fig. 3b, the improvement indicates that the rate of new proteins with at least one correct prediction generally decreases as a function of $\mathrm{T}_{\mathrm{np}}$. Here, the random expectation describes the percentage of proteins with at least one correct again assuming that all sites are equally probable to be catalytic.

Fig. 4a tabulates the number of proteins observed with a specific number of correct catalytic residue predictions at $\mathrm{T}_{\mathrm{np}}=5$. By far, the most common incidence is zero correct $(76 \%)$, which corresponds to every prediction within a given protein being incorrect. Approximately $17 \%$ of the predictions from the SCOP superfamily parsed dataset have one correct and 7\% have two correct. Less than $1 \%$ has three correct, and none have four or five correct. Again, there is good agreement between the three datasets, yet (as before) there is a slight accuracy reduction within the smaller datasets.

Throughout this report, we use citrate synthase as an example to discuss the context of the CC results. Citrate synthase is chosen because it nicely demonstrates how the two filters discussed below improve prediction accuracy. Moreover, citrate synthase is an important enzyme in aerobic metabolism; it regulates the pace of the Krebs cycle. The enzyme catalyzes the condensation between the two acetyl carbons from acetyl-CoA and oxaloacetate to form citrate [38]. The reaction is energetically driven by hydrolysis of the thioester bond, which is strongly exothermic, within acetyl-CoA. None of the predictions at $\mathrm{T}_{\mathrm{np}}=5$ correspond to catalytic residues. While we are only narrowly using catalytic residues to benchmark the approach, this lack of sensitivity should not be interpreted as a complete failure to provide useful information. Similar to the exam- 
Table 2: Evaluation of catalytic site predictions.'

\begin{tabular}{|c|c|c|c|c|c|c|c|}
\hline Avg.\#/PDB & Total accuracy ${ }^{2}$ & Per PDB accuracy 3 & p-value ${ }^{4}$ & TP \& FP rate 5 & TP:FP ratio & I correct per PDB ${ }^{6}$ & $\begin{array}{l}\text { I correct } \\
\text { expect }^{7}\end{array}$ \\
\hline \multicolumn{8}{|c|}{ (a.) Raw CC values (no filter) } \\
\hline 1.3 & 6.0 & $2.7(10.8)$ & 2.7E-09 & $2.1 / 0.4$ & 6.0 & 7.6 & 1.1 \\
\hline 2.4 & 6.8 & $4.2(11.6)$ & $2.8 \mathrm{E}-22$ & $4.9 / 0.7$ & 7.2 & 15.0 & 2.0 \\
\hline 3.6 & 6.5 & $4.5(10.6)$ & $2.4 \mathrm{E}-30$ & $7.0 / 1.0$ & 6.9 & 19.9 & 3.1 \\
\hline 4.6 & 6.3 & $4.7(10.0)$ & $2.4 \mathrm{E}-37$ & $8.8 / 1.3$ & 6.9 & 23.4 & 3.9 \\
\hline 5.7 & 6.3 & $4.9(9.6)$ & $9.4 \mathrm{E}-47$ & $11.0 / 1.6$ & 6.9 & 27.6 & 4.8 \\
\hline \multicolumn{8}{|c|}{ (b.) Solvent accessibility filter } \\
\hline 1.1 & 14.2 & $7.5(17.4)$ & $2.8 \mathrm{E}-42$ & $5.3 / 0.3$ & 18.7 & 15.9 & 1.0 \\
\hline 2.2 & 13.0 & $9.2(16.8)$ & 7.5E-72 & $9.7 / 0.6$ & 16.9 & 25.4 & 1.9 \\
\hline 3.3 & II.I & $8.7(14.7)$ & $6.8 \mathrm{E}-82$ & $12.2 / 0.9$ & 14.2 & 29.3 & 2.9 \\
\hline 4.4 & 10.8 & $8.9(13.2)$ & 4.5E- 103 & $15.8 / 1.2$ & 13.7 & 36.7 & 3.9 \\
\hline 5.4 & 10.4 & $8.9(12.4)$ & $2.7 \mathrm{E}-1204$ & $18.8 / 1.4$ & 13.2 & 41.3 & 4.8 \\
\hline \multicolumn{8}{|c|}{ (c.) Residue identify filter } \\
\hline I.I & 22.4 & $11.3(21.0)$ & $3.8 \mathrm{E}-83$ & $8.3 / 0.3$ & 32.6 & 23.0 & 1.0 \\
\hline 2.2 & 19.6 & $13.5(19.8)$ & $8.8 \mathrm{E}-134$ & $14.5 / 0.5$ & 27.6 & 35.7 & 1.9 \\
\hline 3.2 & 17.9 & $13.8(18.2)$ & 0.0 & 19.2/0.8 & 24.7 & 42.8 & 2.8 \\
\hline 4.3 & 17.6 & $14.3(17.0)$ & 0.0 & $25.0 / 1.0$ & 24.1 & 50.5 & 3.7 \\
\hline 5.2 & 16.5 & $13.9(156.3)$ & 0.0 & $29.3 / 1.3$ & 22.4 & 56.2 & 4.7 \\
\hline \multicolumn{8}{|c|}{ (d.) Combination filter (solvent accessibility + resodue identify) } \\
\hline I.I & 25.2 & $12.9(21.8)$ & 0.0 & $18.6 / 0.5$ & 39.0 & 26.1 & 1.0 \\
\hline 2.1 & 20.7 & I $4.4(20.7)$ & 0.0 & $31.0 / 1.0$ & 30.8 & 36.7 & 1.9 \\
\hline 3.1 & 17.9 & $13.5(17.1)$ & 0.0 & $39.9 / 1.5$ & 26.2 & 44.2 & 2.7 \\
\hline 4.1 & 15.9 & $12.8(14.6)$ & 0.0 & $45.4 / 2.1$ & 21.8 & 49.8 & 3.6 \\
\hline 5.2 & 13.9 & $11.7(13.1)$ & 0.0 & $50.0 / 2.7$ & 18.7 & 53.0 & 4.6 \\
\hline
\end{tabular}

\footnotetext{
I Statistics describing the accuracy of the accessibility-filtered prediction on the SCOP superfamily dataset. ${ }^{2}$ Accuracy is defined as the percentage of correct catalytic residue predictions out of the total number of predictions for the entire collapsed dataset. In all cases, the random expectation is $0.9 \%$. ${ }^{3}$ Average value (and standard deviation) of accuracy calculated on a per protein basis. ${ }^{4}$ The probability that the null hypothesis is correct calculated from the binomial distribution. ${ }^{5}$ The true positive rate is the percent correct of the total number of catalytic residues within the CSA; similarly, the false positive rate is the percent incorrect predictions of the total number of noncatalytic residues. ${ }^{6}$ The percent of proteins with at least one correct prediction. ${ }^{7}$ The expected percent of proteins with at least one correct assuming a random model.
}

ples shown in Fig. 1, the five most central residues (Tyr185, Ala186, Phe333, Met335 and Gly336, using 1 AJ8 numbering) are all buried deep with the core of the protein; in fact, four are completely inaccessible to solvent. Despite their location within the core, Tyr185 and Phe333 are both clearly important as they structurally contact the catalytic Asp312. Moreover, Phe333 is also contacting the citrate substrate. While all non-protein (HETERO) groups have been stripped from our inputs to make this large-scale analysis tractable, it is evocative that the model is picking residues directly interacting with the substrate, even if they are not catalytic per se. Below it is demonstrated that filtering CC predictions by residue accessibility and/or residue identity substantially improves citrate synthase catalytic residue prediction accuracy.

\section{Improving prediction accuracy by excluding the most buried residues}

Straightforward physical intuition suggests that the most buried residues within the protein are likely to have the highest CC values. Fig. 1 clearly demonstrates this expectation to be correct. However, conventional wisdom also states that most catalytic residues are (at least partially) exposed to solvent [20]. For example, it is very common to find catalytic residues at the bottom of an active residue cleft where they are partially obscured from solvent. This is because some exposure to solvent is important for recognition by the incoming substrate. Moreover, water molecules are frequently utilized along the reaction coordinate. As such, it makes sense to filter residue completely inaccessible from solvent from further consideration.

As a first step toward improving CC catalytic residue predictions using solvent accessibility, we begin by asking the question, "Are the solvent accessibility distributions of catalytic and noncatalytic residues significantly different?" Additional file 3 clearly shows that the two distributions are very similar. This result justifies the approach because it demonstrates that CC does not simply recapitulate solvent accessibility. Put in other words, CC provides information orthogonal to accessibility. This point is further demonstrated in Additional file 4 that plots accessibility vs. CC for catalytic and noncatalytic residues. Similar to the value reported within Amitai et al. [32], the overall correlation between solvent accessibility and CC is low (R 

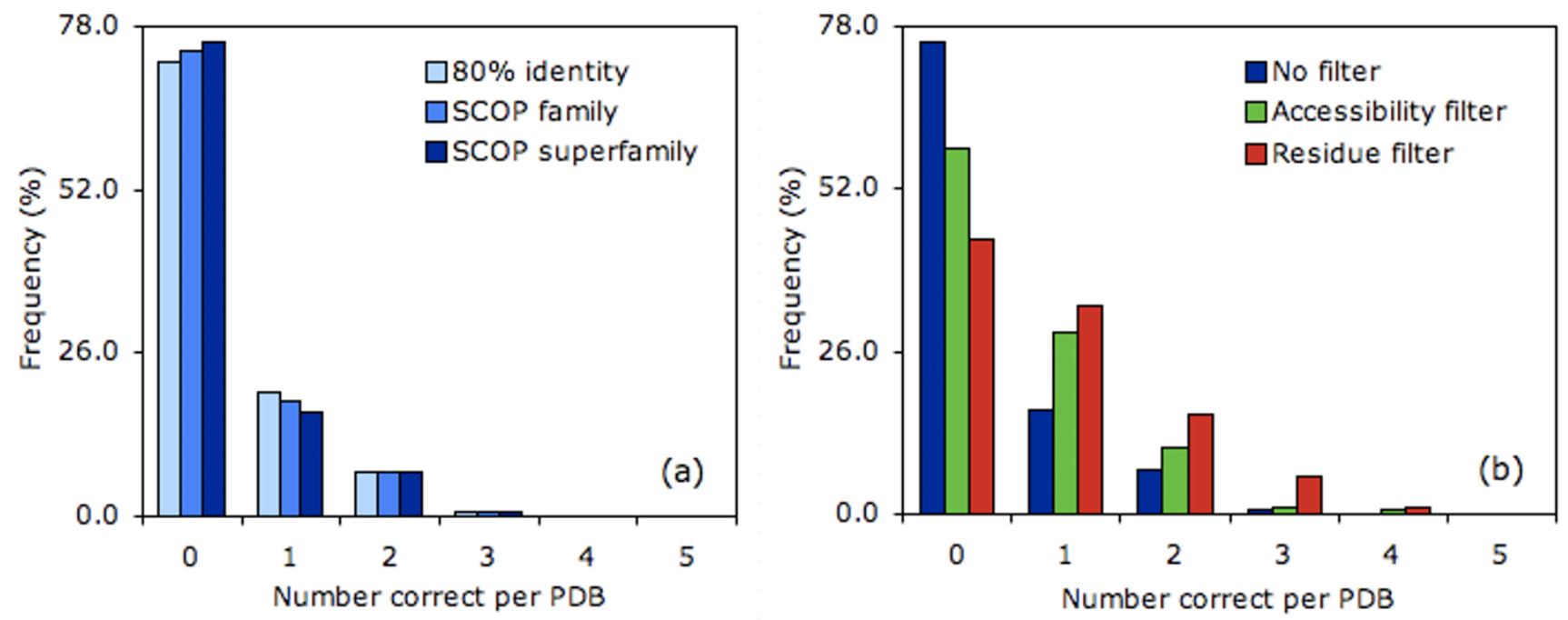

\section{Figure 4}

(a) For $\mathrm{T}_{\mathrm{np}}=5$, the percentage of PDBs with 0-5 correct predictions are tabulated for each of the three datasets investigated. (b) The same data is plotted for each of the three filtering schemes investigated for the SCOP superfamily parsed dataset. Note: the dark blue series in (a) is exactly the same as in (b).

$=-0.28)$. Finally, we use mutual information (MI) to quantify the amount of (in)dependence between the two metrics. The MI between solvent accessibility and CC is 0.011 ; a value of zero indicates complete independence. Consequently, it makes physical sense to combine the two metrics. This would not be the case if closeness centrality simply reflected solvent accessibility.

We introduce the solvent accessibility threshold, $\mathrm{T}_{\mathrm{sa}^{\prime}}$ to filter out residues with low solvent accessibilities. All residues with residue solvent accessibility $<\mathrm{T}_{\mathrm{sa}}$ are a priori excluded as catalytic residue predictions. Additional file 5 shows two example plots of how accessibility filtering improves prediction accuracy. In all cases, any amount of accessibility filtering significantly increases the prediction accuracies. In the $\mathrm{T}_{\mathrm{np}}=2$ example, the maximal relative accuracy occurs at $\mathrm{T}_{\mathrm{sa}}=8 \AA^{2}$, which corresponds to a prediction accuracy of $13.1 \%$. The associated false and true positive rates are $0.6 \%$ and $9.8 \%$, respectively. When $\mathrm{T}_{\mathrm{np}}=$ 5 , the maximal accuracy (10.4\%) occurs at $\mathrm{T}_{\mathrm{sa}}=9 \AA^{2}$. The corresponding false positive rate is $1.4 \%$, and the true positive rate is $18.8 \%$. One might argue that the performance improvement shown here is simply a matter of opening a free parameter with no transferability. In order to test parameter transferability, the parsed dataset was randomly divided into two halves, and the same analysis was performed on each. The resulting ideal thresholds are very close $\left( \pm 1.0 \AA^{2}\right)$ to each other and to the values for whole dataset. This result confirms the transferability of the identified $\mathrm{T}_{\mathrm{sa}}$ values. Using a fixed $\mathrm{T}_{\mathrm{sa}}=9.0 \AA^{2}$, which is the most common best value observed, Table $2 \mathrm{~b}$ tabulates the accuracy of the approach at each $\mathrm{T}_{\mathrm{np}}$. In all cases, the values are greater than the corresponding unfiltered results. Once more, the values from the collapsed and per protein datasets are similar, especially when considering the standard deviation within the per protein values as an error estimate.

Fig. $4 \mathrm{~b}$ plots the percentage of PDBs with $0-5$ correct predictions $\left(\mathrm{T}_{\mathrm{sa}}=8 \AA^{2}\right)$, which further demonstrates that the solvent accessibility filter improves accuracy. Compared to the unfiltered predictions, there are fewer proteins with zero correct predictions, and more with one or two correct. In the parsed dataset, the improvement for one, two, three and four correct is $12.4 \%, 3.5 \%, 0.4 \%$ and $0.7 \%$, respectively. In all cases, the p-value for the accessibilityfiltered predictions is lower than the corresponding unfiltered results (Additional file 2). In fact, in spite of a global reduction in the number of predictions, the $\mathrm{p}$-value of the accessibility-filtered results is lowered by 33 to 73 orders of magnitude.

As before, we briefly discuss the context of the predictions within the citrate synthase example. Here, the improvement within the catalytic residue predictions is stark. Citrate synthase has three catalytic residues annotated within the CSA. These residues (His223, His262 and Asp312) are structurally proximal to each other and reside within the active residue cleft. Each directly interacts with a carboxyl group of the enzyme's citrate substrate. Recall that the predictions based solely on CC are inaccessible to solvent. On the other hand, all three of the enzyme's catalytic res- 
idues are partially exposed to solvent in both the functional dimer and the constituent monomers that are our predictions are based. The monomer exposure of His223, His 262 and Asp 312 is 34,52 and $10 \AA^{2}$, respectively. The accessibilities of His223 and His262 within the dimer are slightly reduced, whereas the Asp312 value is unaffected. Based solely on CC (i.e. no filtering), the network model fails to predict either of the catalytic residues; in fact, they only rank order $27^{\text {th }}, 43^{\text {rd }}$ and $172^{\text {nd }}$ (of 371 residues). Nevertheless, after filtering all residues solvent accessibilities less than $9 \AA^{2}$, His223 and Asp312 are correctly predicted to be catalytic.

As suggested above, sites other than the catalytic residues can also be critical to function [38-40]. Additionally, it is possible that sites not annotated within the CSA might also be catalytic, or at the very least, directly related to functional efficiency. In fact, Russell et al. define ten additional active site residues as being critical to function [37]. In spite of this more liberal definition, none of the remaining three accessibility-filtered predictions (Glu189, Lys219 and Glu228) correspond to sites within the expanded benchmark. Nevertheless, these residues are clearly important, as they are structurally proximal to both catalytic sites. This result is trivially expected due to their sequence proximity to His 218 ; however, the fact that CC, which treats considers each vertex without regard to primary structure, is promising.

\section{Filtering based on residue identity}

While we explicitly avoid alignment and phylogeny data here, it might be possible to improve prediction accuracy by simply filtering out residues that are unlikely to be catalytic based on their innate physiochemical properties. For example, in the neural networkbased prediction approach of Gutteridge et al. [19], it is demonstrated that the single most import element of the input is whether or not the residue being considered is histidine. The second most important element is residue conservation, which is followed closely by whether or not the residue in question is lysine, cysteine, aspartate, glutamate and arginine (in that order). These sequencebased input elements are all more important than a variety of commonsense structural characteristics (i.e. depth, solvent accessibility, cleft information and secondary structure). Consequently, we implement a simple filter based on residue identity here. Any residue that is not histidine, lysine, cysteine, aspartate, glutamate or arginine is excluded from further consideration. We have tried other combinations of residue exclusion, but this provides the best overall results. A comparison of per residue CC values for catalytic and noncatalytic residues is provided in Additional file 6.

The accuracy of the residue identity filtered predictions ranges from 16.5 to $22.4 \%$, which is a substantial improvement over the random expectation of $0.9 \%$ (Table 2c). Predicting catalytic residues by residue identity alone provides a second baseline to compare to. In this approach, a prediction is put forth each time one of the six residue types listed above occurs. Using only residue identity results in an accuracy of $2.1 \%$, which is only slightly better than random expectation of $0.9 \%$. Moreover, it is substantially less than the residue identity filtered results, meaning CC substantially improves predictive power over residue identity alone. Like before, the per protein accuracy range is significantly less (11.3 to $14.3 \%)$ than the collapsed results. Nevertheless, the main result that the method significantly improves upon the solvent accessibility filtered predictions is clearly conserved.

Fig. 5a plots the improvement of the three prediction schemes against $\mathrm{T}_{\mathrm{np}}$. In all cases, the improvement of the residue identity filtered predictions perform substantially better than the other two schemes. Moreover, an improvement is observed both when considering total prediction accuracy (circles) and the percentage of proteins with at least one correct prediction (squares). As expected, the residue identity filtered p-values are also smaller than the accessibility-filtered ones, despite the fact that there are fewer overall residue filtered predictions. In fact, over the first two values of $\mathrm{T}_{\mathrm{np}}$, the improvement is 41 and 62 orders of magnitude, respectively. Due to a lack of floating point precision, $\mathrm{p}$-values for the last three are calculated to be exactly zero. Fig. $4 \mathrm{~b}$ also tabulates the number of PDBs with $0-5$ correct residue identity filtered predictions. Again, there is a greater increase in the nonzero bin compared to the accessibility-filtered results. The increase in the number of correctly identified proteins (compared to the unfiltered results) with one, two, three and four correct is $16.6 \%, 8.8 \%, 5.3 \%$ and $1.1 \%$, respectively. These numbers correspond to an improvement over the accessibility-filtered predictions of $4.2 \%, 5.3 \%, 4.9 \%$ and $0.4 \%$, respectively.

The residue identity filter decides whether to consider or not consider a particular residue type based on an a priori scheme. This is equivalent as saying that the six residues that "pass through" the residue identity filter are equally probable. However, Additional file 6 clearly indicates that this is not reality. As such, it is natural to assume that some sort of fuzzy logic scheme that allows residues to be in the considered or excluded set based on the observed catalytic residue propensities should improve model accuracy. An exhaustive number of schemes were tried using various weighting schemes. For example, three possibilities (from several different considerations) include: (i.) weighting all twenty residues exactly proportional to their catalytic propensity; (ii.) weighting the six from above as equally probable, but scaling of the others; and (iii.) weighting the six from above with exclusion of the 

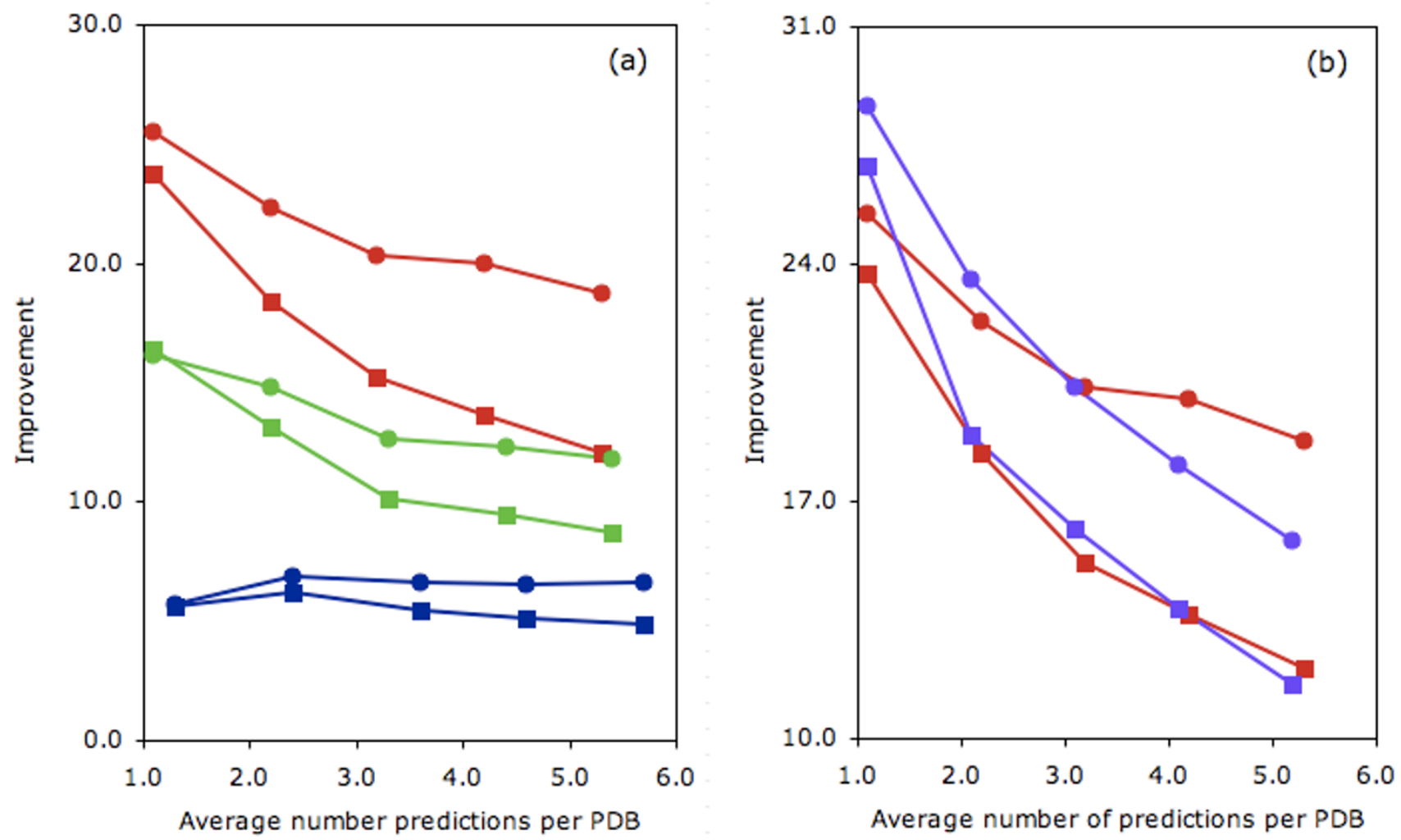

\section{Figure 5}

(a) Improvement for all predictions and percent of proteins with at least one correct is plotted for: no filtering (blue), solvent accessibility filtering (green) and residue identity filtering (red). The data presented is from the SCOP superfamily parsed dataset. Symbols are the same as in Fig. 3. In (b), the combination (accessibility + residue identity) filter (magenta) is shown to perform very similar to the residue identity filter alone.

remainder. However, no statistically significant improvement over what is reported in Table $2 \mathrm{c}$ is found. In the first two examples, the fuzzy model actually does worse since catalytic residues make up such a tiny fraction of the total number of residues. Meaning, any relaxed filtering criteria allows many more noncatalytic (vs. catalytic) residues to be considered; consequently, specificity is lost. Conversely, the best of the trials within the third scheme is statistically indistinguishable from Table $2 \mathrm{c}$.

Filtering based on residue identity results in the following set of predictions (Glu187, His188, Lys219, Asp312 and Arg337) within citrate synthase at $\mathrm{T}_{\mathrm{np}}=5$. Asp312 and Lys 219 are discussed above, whereas Glu187, His 188 and Arg337 are noncatalytic residues within the active site region. As with Phe333, both His188 and Arg337 are directly interacting with the citrate substrate (see Fig. 6). These residues are also interacting with several of the residues identified without filtering. This result, along with those from above, highlights the fact that the three different prediction schemes are identifying citrate synthase residues structurally proximal to the catalytic residues. A similar overall trend is observed when investigating other proteins.

\section{Combining solvent accessibility and residue identity}

Combining both filters together results in slight improvement over the residue identity filter at $\mathrm{T}_{\mathrm{np}}=1-2$ (Fig. 5b). At $\mathrm{T}_{\mathrm{np}}=4$ and 5 , the combination slightly underperforms the residue identity filter, yet the values are still significantly better than the accessibility-filtered results. (At $\mathrm{T}_{\mathrm{np}}$ $=3$ the results are virtually identical to the residue identity filtered predictions.) The likely explanation for this result is due to the fact that the filters eliminate similar information. For example, it is trivially expected, due to their propensity to be within the core, that residues eliminated by the accessibility filter will be nonpolar amino acids. Likewise, the residue identity filter always eliminates nonpolar residues from further consideration. As done above with the residue identity filtered results, a baseline without CC is considered. In this instance, any time one of the six considered residue types occurs with a solvent accessibility below $9 \AA^{2}$, a prediction is put forth. This scheme results in an accuracy of $2.5 \%$, slightly better than the $2.1 \%$ of 


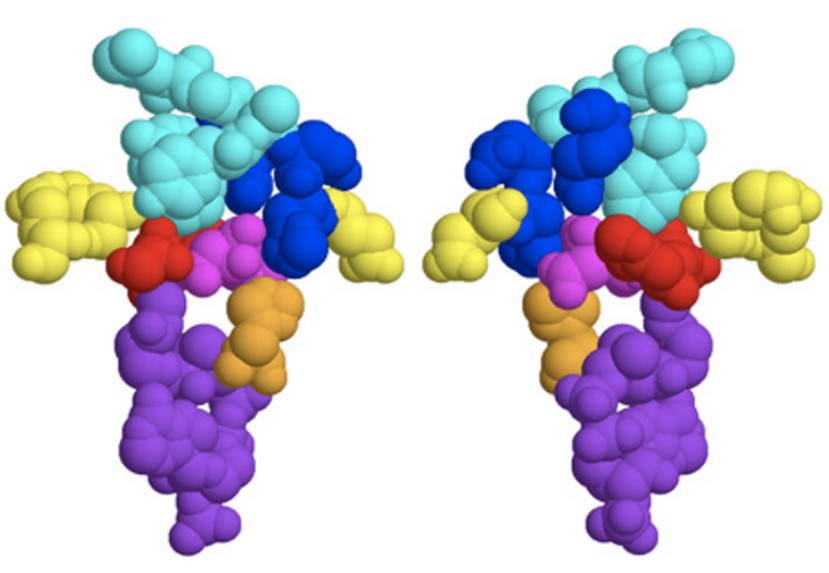

Figure 6

All predictions within citrate synthase, as discussed in the text, are shown in spacefill. Note that they all cluster within the active site region. Coenzyme-A and the citrate substrate are shown in purple and magenta, respectively. The two correctly predicted catalytic residues are shown in red; the unpredicted catalytic residue (His262) is shown in orange. The image on the left, which is rotated 180 degrees from the one on the right, is centered on Asp 312 whereas the image on the right is centered on His223. Unfiltered predictions are colored cyan. Except for Lys219, the remaining accessibility and residue identity filtered predictions are colored yellow and blue, respectively. Lys 219 , which is predicted by both filtering schemes, is colored green (yellow + blue = green).

residue identity alone, yet nowhere near the accuracies of the combination-filtered CC scores.

\section{Sensitivity to structural input}

It is also important to assess the sensitivity of the CC method to structural input variations. Specifically, ligation state could have a pronounced affect on the observed results. To explore the effects of bound substrate, thirteen randomly chosen structure pairs (with and without ligand) are compared (Table 3). The chosen structures represent a diverse spectrum of protein sizes and SCOP classes. Encouragingly, the average correlation coefficient between CC values for each pair is very high $(<R>=0.970$; standard deviation $=0.049$ ), meaning that CC is rather robust to the structural differences. Surprisingly, there is no correlation between CC correlation and pairwise structural RMSD calculated via combinatorial extension [40] (see Table 3). It is also important to consider what is happening between specific catalytic pairs. Comparing the relative rank ordering within each catalytic residue pair reveals that the rank within ligated structures increases $46.2 \%$ of the time. Conversely, the rank increases $41.0 \%$ of the time within unligated structures; there is no change in the rank $12.8 \%$ of the time. Accordingly, there is no systematic performance increase when choosing structures based on the presence or absence of bound substrate.
Similarly, we also examined the sensitivity of the CC method to slight structural perturbations. Here, we use molecular dynamics simulations (MD) to "shake up" the structure a small amount (we specifically focus on slight structural perturbations) and compare the resultant CC values. Additional file 7 plots the average $\mathrm{CC}$ value + standard deviation for the citrate synthase conformers. The plot clearly demonstrates that CC is fairly robust to these slight structural rearrangements. More importantly, the results concerning catalytic sites predicted after filtering results are overall unchanged. Fig. 7 plots the CC standard deviation vs. the CC rank for each residue within citrate synthase. Residues at the extremes of the CC distribution vary little across the simulation; whereas, residues with CC values near the norm fluctuate much more. Similar results are observed in three other MD simulations. Since catalytic residue CC values are not exclusively at the top end of the distribution, the potential for slight fluctuations to affect prediction accuracy is present (even though it is not observed here). Future work will explore this result more thoroughly.

\section{Conclusion}

This report investigates the ability of CC to predict enzyme catalytic residues from topological descriptions of protein structure. While the most central residues generally correspond to positions within the core, the predictions are substantially better than the random expectation. This result is maintained whether one averages over the collapsed or per protein datasets. Filtering the predictions by solvent accessibility and/or residue identity improves the results considerably. Overall, these results are comparable to those from previous reports [32,33], but have better statistics due to database size and composition. Additionally, we carefully examine the effect of input structure on our predictions. Pairwise comparisons between ligated and unligated structures reveals no clear trend regarding which input is a better choice. Similarly, slight structural perturbations of four protein examples via MD simulation have no observed effect on the overall conclusions.

\section{Methods \\ Dataset}

Three different datasets extracted from the manually annotated CSA entries are examined here. The first, which contains 568 PDB files, represents a dataset randomly culled such that no two sequences have greater than $80 \%$ sequence identity. The second and third datasets use structural information to randomly distil to nonredundant SCOP [36] families (423 proteins) and superfamilies (283 proteins). In each dataset, a single chain per protein structure is included; however, our analysis of all chains demonstrates that the overall accuracies are generally robust to chain differences (results not shown). All figures shown 
Table 3: Dataset used in comparison of ligated and unligated pairs

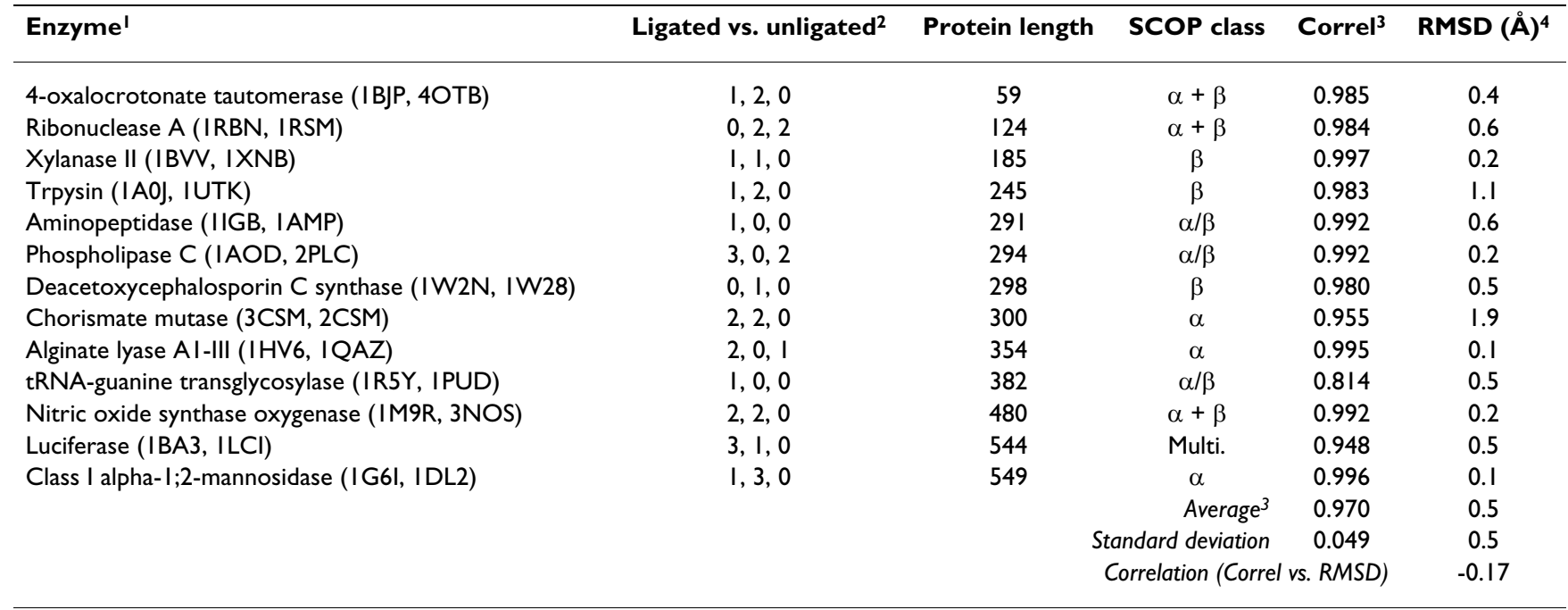

\footnotetext{
I SCOP protein name for each pair examined. The ligated and unligated PDB id's, respectively, are provided in parentheses. ${ }^{2}$ The three values (ligated, unligated, tie) tabulate the number of catalytic residues with higher rank ordering between the structural pair. ${ }^{3}$ Linear correlation coefficient comparing the CC scores between each structural pair. ${ }^{4}$ Pairwise RMSD comparing structure similarity within each pair. Surprisingly, there is no significant correlation between the last two columns.
}

herein are based on the dataset parsed by SCOP superfamily. However, results for the other two datasets are always similar. This point is typified by Fig. 3 and Fig. 4a, which include data for all three.

\section{Solvent accessibility}

We test the ability of solvent accessibility to improve prediction accuracy by filtering out the most buried residues. Solvent accessibility is calculated using DSSP [42], which is an extremely fast approach. DSSP calculated solvent accessibilities range between 0 to $>250 \AA^{2}$. No percent or relative accessibility corrections, which are commonly employed to normalize values by sidechain surface area

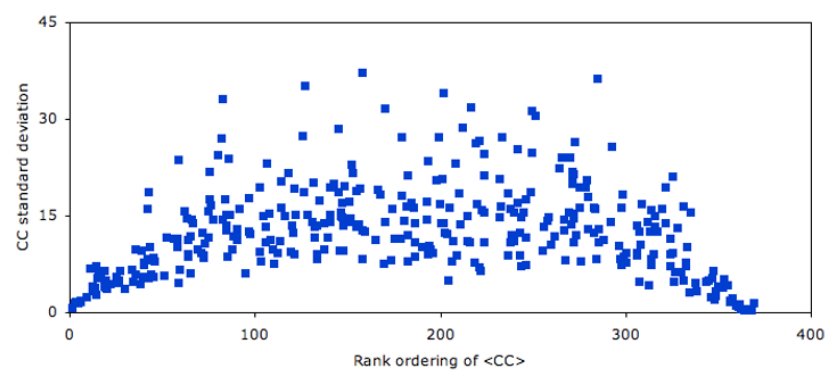

\section{Figure 7}

The standard deviation within the CC scores across the structural ensemble is plotted against the average rank ordering of each position for citrate synthase. Residues at the extreme ends of the rank ordering are rather insensitive to the structural variations; however, residues nearer the norm vary significantly. Similar plots are observed in simulations of acetate kinase, triosephosphate isomerase and malate dehydrogenase. and to remove backbone considerations, are implemented within DSSP. Nevertheless, the lack of these corrections is not critical here as we are simply trying to identify the residues most excluded from solvent. Theses corrections are more important when quantifying solvent exposure because the maximal accessibility of a large residue (i.e. lysine) is so much greater than that of a small residue (i.e. alanine). Conversely, in our problem, if both residues are maximally buried, the accessibility (with or without the correction) is simply zero in each case.

\section{Molecular dynamics}

Molecular dynamics simulations are employed to generate an ensemble of slightly perturbed structures. The protocol used here is the same as we reported previously in our analysis of sensitivity within calculated pKa values [43]. Canonical ensemble (fixed NVT) in vacuo molecular dynamics simulations, as implemented in the Molecular Operating Environment (Chemical Computing Group, Montreal, Quebec, Canada), are used to generate the ensemble of conformers. In each example, the timescale of the simulations is $1 \mathrm{~ns}$, and the timestep is $0.001 \mathrm{ps}$. Structure sampling occurs every 500 ps. It is obvious that this in vacuo simulation protocol is unacceptable to determine realistic aqueous phase dynamics. However, it is adequate for the aims of this work since the simulation is simply used to generate a conformational distribution.

\section{Abbreviations}

Evolutionary trace (ET); Catalytic site atlas (CSA); Closeness centrality (CC); Probability density function (PDF); Receiver operating characteristic (ROC); Mutual information (MI); Molecular dynamics (MD). 


\section{Authors' contributions}

EC and DRL contributed equally to the performance of the work described herein. DRL oversaw the research and wrote the manuscript. Both authors have read and approved the final version of the manuscript.

\section{Additional material}

\section{Additional file 1}

Supplementary figure 1. This file contains an example ROC curve.

Click here for file

[http://www.biomedcentral.com/content/supplementary/1471-

2105-8-153-S1.pdf]

\section{Additional file 2}

Supplementary figure 2. The probability of the null hypothesis being correct at each $T_{n p}$ value.

Click here for file

[http://www.biomedcentral.com/content/supplementary/14712105-8-153-S2.pdf]

\section{Additional file 3}

Supplementary figure 3. Probability density functions of the solvent accessibility scores for all catalytic and all noncatalytic sites.

Click here for file

[http://www.biomedcentral.com/content/supplementary/14712105-8-153-S3.pdf]

\section{Additional file 4}

Supplementary figure 4. Scatter plot of residue solvent accessibility vs. closeness centrality.

Click here for file

[http://www.biomedcentral.com/content/supplementary/14712105-8-153-S4.pdf]

\section{Additional file 5}

Supplementary figure 5. The relative accuracy vs. solvent accessibility thresholds is plotted.

Click here for file

[http://www.biomedcentral.com/content/supplementary/1471-

2105-8-153-S5.pdf]

\section{Additional file 6}

Supplementary figure 6. Histogram comparing the catalytic vs. noncatalytic average closeness centrality values for each residue type.

Click here for file

[http://www.biomedcentral.com/content/supplementary/1471-

2105-8-153-S6.pdf]

\section{Additional file 7}

Supplementary figure 7. Average CC value vs. sequence position for citrate synthase.

Click here for file

[http://www.biomedcentral.com/content/supplementary/14712105-8-153-S7.pdf]

\section{Acknowledgements}

Andrei Istomin is thanked for proof reading the manuscript. Anthony Fodor is thanked for reading an early draft of this paper and providing several valuable suggestions. The reviewers are also thanked for a number of helpful suggestions. Swati Pande is thanked for constructing the ROC plots. James Torrance, from Janet Thornton's research group, is thanked for assistance with the Catalytic Site Atlas. This work is supported by a Joint Ventures Grant to DRL from the California State Program for Education and Research in Biotechnology. DRL is supported, in part, by NIH ROI GM073082. EC is supported by Howard Hughes Medical Institute undergraduate fellowship.

\section{References}

I. Watson JD, Laskowski RA, Thornton JM: Predicting protein function from sequence and structural data. Curr Opin Struct Biol 2005, I 5(3):275-284.

2. Pazos F, Bang JW: Computational Prediction of Functionally Important Regions in Proteins. Curr Bioinformatics 2006, I:15-23.

3. Jones S, Thornton JM: Searching for functional sites in protein structures. Curr Opin Chem Biol 2004, 8( I):3-7.

4. Innis CA, Anand AP, Sowdhamini R: Prediction of functional sites in proteins using conserved functional group analysis. J Mol Biol 2004, 337(4): 1053-1068.

5. Panchenko AR, Kondrashov F, Bryant S: Prediction of functional sites by analysis of sequence and structure conservation. Protein Sci 2004, I3(4):884-892.

6. Soyer OS, Goldstein RA: Predicting functional sites in proteins: site-specific evolutionary models and their application to neurotransmitter transporters. J Mol Biol 2004, 339(I):227-242.

7. Lichtarge O, Bourne HR, Cohen FE: An evolutionary trace method defines binding surfaces common to protein families. J Mol Biol I996, 257(2):342-358.

8. Aloy P, Querol E, Aviles FX, Sternberg MJ: Automated structurebased prediction of functional sites in proteins: applications to assessing the validity of inheriting protein function from homology in genome annotation and to protein docking. Mol Biol 200I, 3 I I (2):395-408.

9. Madabushi S, Yao H, Marsh M, Kristensen DM, Philippi A, Sowa ME, Lichtarge O: Structural clusters of evolutionary trace residues are statistically significant and common in proteins. J Mol Biol 2002, 316(1):139-154.

10. Yao H, Kristensen DM, Mihalek I, Sowa ME, Shaw C, Kimmel M, Kavraki L, Lichtarge O: An accurate, sensitive, and scalable method to identify functional sites in protein structures. I Mol Biol 2003, 326(I):255-26I.

11. Armon A, Graur D, Ben Tal N: ConSurf: an algorithmic tool for the identification of functional regions in proteins by surface mapping of phylogenetic information. J Mol Biol 200I, 307(I):447-463.

12. Bickel PJ, Kechris KJ, Spector PC, Wedemayer GJ, Glazer AN: Finding important sites in protein sequences. Proc Natl Acad Sci USA 2002, 99(23): | 4764-|477|.

13. del Sol MA, Pazos F, Valencia A: Automatic methods for predicting functionally important residues. I $\mathrm{Mol}$ Biol 2003, 326(4): $1289-1302$.

14. Landgraf R, Xenarios I, Eisenberg D: Three-dimensional cluster analysis identifies interfaces and functional residue clusters in proteins. J Mol Biol 200I, 307(5): I487-I502.

15. La D, Livesay DR: MINER: Software for phylogenetic motif identification. Nucleic Acids Res 2005:W267-W270.

16. La D, Livesay DR: Predicting functional sites with an automated algorithm suitable for heterogeneous datasets. $B M C$ Bioinformatics 2005, 6(I): I I6.

17. La $D$, Sutch $B$, Livesay DR: Predicting protein functional sites with phylogenetic motifs. Proteins 2005, 58(2):309-320.

18. Pazos F, Rausell A, Valencia A: Phylogeny-independent detection of functional residues. Bioinformatics 2006, 22(I 2): I440-I 448.

19. Gutteridge A, Bartlett GJ, Thornton JM: Using a neural network and spatial clustering to predict the location of active sites in enzymes. J Mol Biol 2003, 330(4):71 9-734.

20. Bartlett G], Porter CT, Borkakoti N, Thornton JM: Analysis of catalytic residues in enzyme active sites. J Mol Biol 2002, 324(I): $105-121$. 
21. Porter CT, Bartlett G], Thornton JM: The Catalytic Site Atlas: a resource of catalytic sites and residues identified in enzymes using structural data. Nucleic Acids Res 2004:D I29-DI 33.

22. Neves-Petersen MT, Petersen SB: Protein electrostatics: a review of the equations and methods used to model electrostatic equations in biomolecules - applications in biotechnology. Biotechnol Annu Rev 2003, 9:3 15-395.

23. Elcock $\mathrm{AH}$ : Prediction of functionally important residues based solely on the computed energetics of protein structure. J Mol Biol 200I, 3 I 2(4):885-896.

24. Cheng G, Qian B, Samudrala R, Baker D: Improvement in protein functional site prediction by distinguishing structural and functional constraints on protein family evolution using computational design. Nucleic Acids Res 2005, 33( I8):586I-5867.

25. Ondrechen MJ, Clifton JG, Ringe D: THEMATICS: a simple computational predictor of enzyme function from structure. Proc Natl Acad Sci USA 200I, 98(22): I 2473-I2478.

26. Livesay DR, Jambeck P, Rojnuckarin A, Subramaniam S: Conservation of electrostatic properties within enzyme families and superfamilies. Biochemistry 2003, 42(I 2):3464-3473.

27. Livesay $D R$, La $D$ : The evolutionary origins and catalytic importance of conserved electrostatic networks within TIM-barrel proteins. Protein Sci 2005, I4(5): I I58-I I 70.

28. Brinda KV, Vishveshwara S: A network representation of protein structures: implications for protein stability. Biophys 2005, 89(6):4I59-4I70.

29. Dokholyan NV, Li L, Ding F, Shakhnovich El: Topological determinants of protein folding. Proc Natl Acad Sci USA 2002, 99(I3):8637-864I.

30. Greene LH, Higman VA: Uncovering network systems within protein structures. J Mol Biol 2003, 334(4):78I-79I.

31. Vendruscolo M, Dokholyan NV, Paci E, Karplus M: Small-world view of the amino acids that play a key role in protein folding. Phys Rev E Stat Nonlin Soft Matter Phys 2002, 65(6 Pt I):061910.

32. Amitai G, Shemesh A, Sitbon E, Shklar M, Netanely D, Venger I, Pietrokovski S: Network analysis of protein structures identifies functional residues. I Mol Biol 2004, 344(4): I I 35-identity filtered

33. Thibert $B$, Bredesen $D E$, del Rio G: Improved prediction of critical residues for protein function based on network and phylogenetic analyses. BMC Bioinformatics 2005, 6:213.

34. Wangikar PP, Tendulkar AV, Ramya S, Mali DN, Sarawagi S: Functional sites in protein families uncovered via an objective and automated graph theoretic approach. J Mol Biol 2003, 326(3):955-978.

35. del Sol A, Fujihashi H, Amoros D, Nussinov R: Residue centrality, functionally important residues, and active site shape: analysis of enzyme and non-enzyme families. Protein Sci 2006, I5(9):2|20-2| 28

36. Murzin AG, Brenner SE, Hubbard T, Chothia C: SCOP: a structural classification of proteins database for the investigation of sequences and structures. I Mol Biol 1995, 247:536-540.

37. Kabsch W, Sander C: Dictionary of protein secondary structure: pattern recognition of hydrogen-bonded and geometrical features. Biopolymers 1983, 22(1 2):2577-2637.

38. Russell RJ, Ferguson JM, Hough DW, Danson MJ, Taylor GL: The crystal structure of citrate synthase from the hyperthermophilic archaeon pyrococcus furiosus at $1.9 \mathrm{~A}$ resolution. Biochemistry 1997, 36(33):9983-9994.

39. Usher KC, Remington S], Martin DP, Drueckhammer DG: A very short hydrogen bond provides only moderate stabilization of an enzyme-inhibitor complex of citrate synthase. Biochemistry 1994, 33(25):7753-7759.

40. Alter GM, Casazza JP, Zhi W, Nemeth P, Srere PA, Evans CT: Mutation of essential catalytic residues in pig citrate synthase. Biochemistry 1990, 29(33):7557-7563.

41. Kurz LC, Shah S, Frieden C, Nakra T, Stein RE, Drysdale GR, Evans CT, Srere PA: Catalytic strategy of citrate synthase: subunit interactions revealed as a consequence of a single amino acid change in the oxaloacetate binding site. Biochemistry 1995 34(4I): I3278-I3288.

42. Livesay DR, Jacobs DJ, Kanjanapangka J, Chea E, Cortez H, Garcia J, Kidd P, Marquez MP, Pande S, Yang D: Probing the conformational dependence of calculated pKa values. J Chem Theor \& Comp 2006, 2(4):927-938.
Publish with Bio Med Central and every scientist can read your work free of charge

"BioMed Central will be the most significant development for disseminating the results of biomedical research in our lifetime. "

Sir Paul Nurse, Cancer Research UK

Your research papers will be:

- available free of charge to the entire biomedical community

- peer reviewed and published immediately upon acceptance

- cited in PubMed and archived on PubMed Central

- yours - you keep the copyright
BioMedcentral 